\title{
Killing cells by targeting mitosis
}

\author{
E Manchado ${ }^{1}$, M Guillamot $^{1}$ and M Malumbres ${ }^{*, 1}$
}

Cell cycle deregulation is a common feature of human cancer. Tumor cells accumulate mutations that result in unscheduled proliferation, genomic instability and chromosomal instability. Several therapeutic strategies have been proposed for targeting the cell division cycle in cancer. Whereas inhibiting the initial phases of the cell cycle is likely to generate viable quiescent cells, targeting mitosis offers several possibilities for killing cancer cells. Microtubule poisons have proved efficacy in the clinic against a broad range of malignancies, and novel targeted strategies are now evaluating the inhibition of critical activities, such as cyclin-dependent kinase 1, Aurora or Polo kinases or spindle kinesins. Abrogation of the mitotic checkpoint or targeting the energetic or proteotoxic stress of aneuploid or chromosomally instable cells may also provide further benefits by inducing lethal levels of instability. Although cancer cells may display different responses to these treatments, recent data suggest that targeting mitotic exit by inhibiting the anaphase-promoting complex generates metaphase cells that invariably die in mitosis. As the efficacy of cell-cycle targeting approaches has been limited so far, further understanding of the molecular pathways modulating mitotic cell death will be required to move forward these new proposals to the clinic.

Cell Death and Differentiation (2012) 19, 369-377; doi:10.1038/cdd.2011.197; published online 6 January 2012

\section{Facts}

- Extensive understanding of cell cycle regulation has led to the proposal of new strategies for cancer therapy.

- Targeting regulators of centrosome duplication and separation, spindle formation and mitotic checkpoint have demonstrated efficacy in preclinical models but limited activity in clinical trials.

- The balance between mitotic cell death pathways and kinetics of cyclin B1 degradation determines the therapeutic effectiveness of antimitotic-based strategies.

- Targeting mitotic exit has been proposed as a better therapeutic strategy than targeting spindle assembly and spindle regulators.

- Sustained mitotic arrest leads to a poorly characterized cell death, which may be modulated by cyclin-dependent kinase 1 (Cdk1) activity.

\section{Open Questions}

- Why new mitotic inhibitors have demonstrated limited activity in vivo in comparison with classical microtubuletargeting drugs?

- Newly proposed strategies need to be validated in vivo before reaching clinical settings.

- We need to understand in detail the molecular mechanisms that govern mitotic cell death. Can modulation of these cell death pathways synergize with current antimitotic drugs?
- We need to identify and characterize specific biomarkers for a better definition of patients that may benefit from therapeutic strategies targeting mitosis.

The cell cycle is deregulated in most cancer cells, and multiple strategies have been proposed in the last years to impair tumor cell proliferation. Initial strategies were designed to inhibit the machinery that drives the entry into the cell cycle (G1 phase) and DNA synthesis (S phase). The discovery of centrosomal and mitotic regulators that function during $S$ phase, G2 (a preparatory phase for mitosis (M)) or M phase later provided additional targets, such as mitotic kinases or the mitotic spindle machinery, required for chromosome segregation. The promise of these targets was reinforced by the clinical success of microtubule poisons such as Vinca alkaloids or taxanes. However, targeted therapies directed against the cell cycle have shown limited clinical effect so far. A crucial question in these studies is to what extent targeting the cell cycle merely results in proliferative arrest or induces tumor cell death, and which are the major resistance mechanisms. Recent studies suggest that delaying mitosis or preventing mitotic exit may be highly efficient in killing tumor cells. We will review here current strategies to kill proliferating cells, with specific focus on targeting mitosis or mitotic exit.

\section{Cell Cycle Entry}

Progression through the mammalian cell cycle is driven by several enzymatic activities including protein kinases.

\footnotetext{
${ }^{1}$ Cell Division and Cancer Group, Spanish National Cancer Research Center (CNIO), Madrid, Spain

${ }^{*}$ Corresponding author: M. Malumbres, Centro Nacional de Investigaciones Oncológicas (CNIO), Melchor Fernández Almagro 3, E-28029 Madrid, Spain.

Tel: + 3491 7328000; Fax + 3491 7328033; E-mail: malumbres@cnio.es

Keywords: anaphase-promoting complex-Cdc20; apoptosis; cancer therapy; mitosis; mitotic cell death; mitotic kinases

Abbreviations: APC/C, anaphase-promoting complex/cyclosome; Cdk, cyclin-dependent kinase; CIN, chromosomal instability; PARP, poly(ADP-ribose) polymerase;

RNAi, RNA interference; SAC, spindle assembly checkpoint; SCF, Skp-Cullin-F-box protein complex

Received 11.8.11; revised 30.11.11; accepted 01.12.11; Edited by G Melino; published online 06.1.12
} 
Among them, Cdks are heterodimeric protein kinases composed of a catalytical subunit, called Cdk, and a regulatory subunit, known as cyclin. These complexes are activated sequentially to drive cells through the cell cycle. Whereas Cdk2, Cdk4 and Cdk6 trigger G1/S transition, Cdk1 promotes mitotic entry. ${ }^{1}$ On the one hand, the activity of Cdks is frequently deregulated in tumor cells due to epigenetic or genetic alterations of $\mathrm{Cdk}$-cyclin complexes or to downregulation of several Cdk inhibitors. ${ }^{2}$ Most human tumors display alterations in these regulators, and interphase Cdks were soon identified as possible anticancer targets. ${ }^{2,3}$ However, the first generation of pan-Cdk inhibitors, such us flavopiridol and UCN-01, did not show clinical advantages due to its side effects. ${ }^{4}$ The analysis of gene-targeted mouse models indicated that Cdk2, Cdk4 and Cdk6 are only essential for the proliferation of some specialized cells, suggesting that Cdk inhibitors are likely to produce certain toxicities by affecting specific cells. ${ }^{3,5-7}$ Nonetheless, Cdk4 inhibition may be effective to prevent Myc-induced skin tumors, ${ }^{8}$ Rasinduced breast cancer ${ }^{9}$ or K-Ras-induced non-small-cell lung carcinoma. ${ }^{10}$ These results suggest that $\mathrm{Cdk}$ inhibition may be exploited in clinical settings taking into consideration the cellular context of the tumor and the pathogenic spectrum of their mutations.

Mechanistically, inhibition of cell cycle entry (e.g., by inhibiting interphase Cdks) is likely to induce cell cycle arrest or quiescence but not apoptosis. The use of small-molecule inhibitors for interphase Cdks may result in certain levels of apoptosis, although the lack of specificity of these compounds prevents any detailed molecular conclusion. ${ }^{2}$ Interfering with the $\mathrm{G} 1 / \mathrm{S}$ machinery generally prevents DNA replication, thus rendering diploid, interphasic cells that cannot proliferate but are viable. For instance, inhibition of Cdk4 prevents the development of lung tumors induced by K-Ras oncogene by triggering senescence, a permanent arrest in G0/G1. ${ }^{10}$ Current data suggest that senescent cells may be cleared by an innate immune response, but the extent to which this may contribute to prevention of age-related pathologies and cancer is currently not clear.

\section{Targeting Mitotic Entry}

In contrast to interphase Cdks, Cdk1 is essential for the mammalian cell cycle, and inhibition of Cdk1 prevents entry into mitosis and arrest cells in $\mathrm{G} 2^{11}$ (Figure 1). Cdk1 binds to A- or B-type cyclins and phosphorylates more than 70 substrates to promote centrosome separation, chromosome condensation, nuclear envelope breakdown and mitotic entry. ${ }^{1}$ Strong inhibition of $\mathrm{Cdk} 1$ is therefore likely to be toxic for normal cells, preventing useful therapeutic concentrations. ${ }^{3}$ However, recent studies suggest the possible use of Cdk1 inhibitors in specific cellular settings. Cdk1 is implicated in DNA repair by homologous recombination, and it is essential for efficient formation of BRCA1 foci. Inhibition of poly(ADP-ribose) polymerase (PARP) results in increased dependency on homologous recombination for DNA repair, and is synthetically lethal with mutations in genes involved in this type of DNA repair such as BRCA. ${ }^{12}$ Partial inhibition of Cdk1 may therefore sensitize BRCA-proficient cancer cells to inhibit PARP, suggesting specific applications of Cdk-targeting compounds in cancer cells. ${ }^{13}$ Similar synthetic lethal interactions are likely to exist in other molecular pathways including combinational inhibition with GSK3 $\beta{ }^{14}$ or inhibition of Cdk1 in Myc-overexpressing tumors. ${ }^{15}$

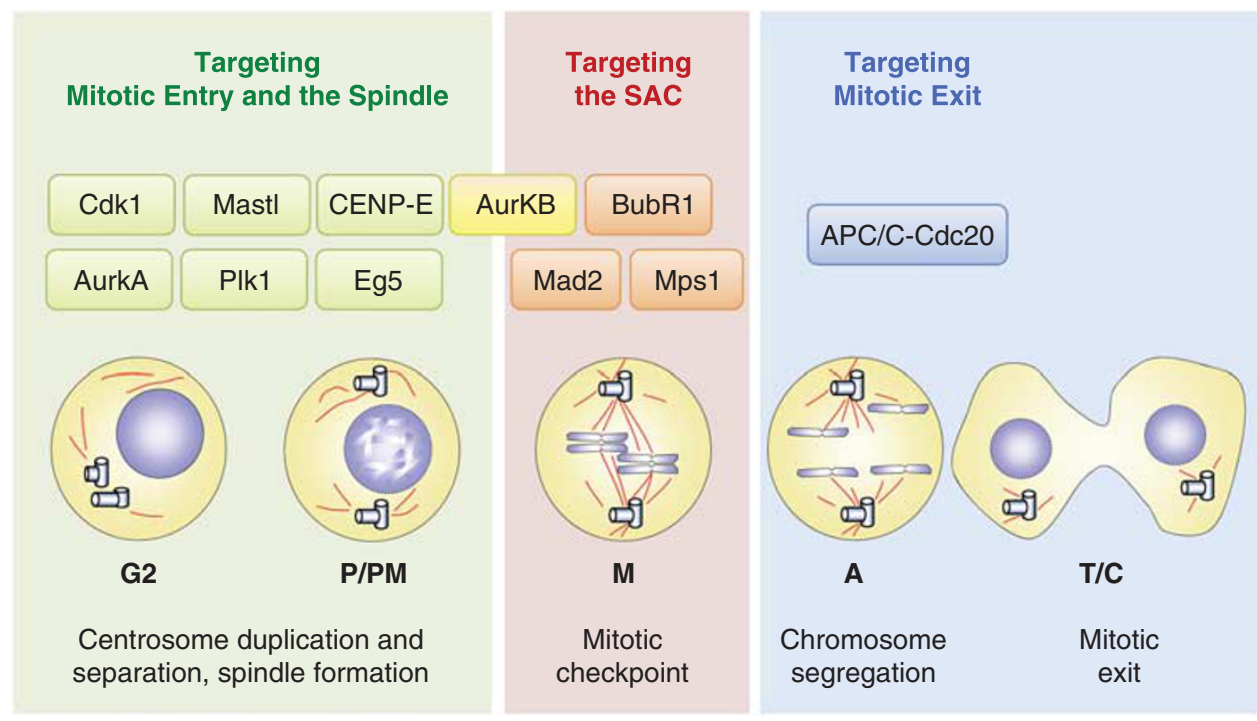

Figure 1 Targeting mitosis for cancer therapy. Cdk1 is a master protein kinase required for several processes during mitotic entry and its inhibition results in G2 arrest. This may require the cooperation of Mastl to inhibit phosphatases, although the relevance of this protein in cancer remains mostly unexplored. Centrosome duplication and separation, and the formation of a bipolar spindle are also required for normal chromosome segregation. Kinases such as Aurora A and Plk1 and kinesins such as Eg5 are critical regulators of these processes and their inhibition results in arrest during prometaphase (PM). This arrest is mediated by the SAC, whose activity depends on several effectors such as Mad2, BubR1, Mps1 or Aurora B, a kinase essential during the error correction mechanism that monitors the proper attachment of microtubules to kinetochores. Abrogation of this checkpoint provokes abnormal chromosome segregation and chromosome instability. APC/C along with its cofactor Cdc20 is required for anaphase (A) onset by targeting critical mitotic regulators for ubiquitin-dependent degradation. Inhibition of this E3-ubiquitin ligase leads to metaphase (M) blockade due to the stabilization of cyclin B1 and mitotic cell death. C, cytokinesis; P, prophase; T, telophase 
In addition to Cdk1, few other targets exist whose inhibition may arrest cells in G2. Mastl, also known as Greatwall in flies and Xenopus, is a protein kinase required for inhibiting PP2A phosphatases that dephosphorylate Cdk substrates. ${ }^{16,17}$ Knockdown of Mastl results in G2 arrest and a variety of mitotic defects, and synergizes with Cdk1 inhibitors, suggesting that both kinase cooperate during mitotic entry. ${ }^{18,19}$ Mastl may be an interesting target for cancer therapy, although the biology of this kinase remains to be studied and no specific inhibitors are available to date.

\section{Targeting the Spindle Assembly}

Microtubule poisons. Mitotic drugs currently used in the clinic are microtubule poisons that perturb microtubule dynamics, impairing the formation of a proper bipolar spindle. Microtubule drugs are usually classified in two main groups: microtubule-destabilizing agents that inhibit microtubule polymerization and microtubule-stabilizing agents that enhance microtubule polymerization. ${ }^{20}$ Vinca alkaloids, such as vinblastine, vincristine, vinorelbine, vindesine and vinflunine, belong to destabilizing compounds and have shown clinical efficacy against a broad range of hematological malignancies. On the other hand, taxanes, such as docetaxel or paclitaxel, are microtubule-stabilizing drugs that are now widely used to treat breast and ovarian tumors, non-small-cell lung cancer and Kaposi's sarcoma. ${ }^{21}$ Both classes of drugs inhibit microtubule dynamics, impairing a functional spindle. This situation is monitored by a mitotic checkpoint, known as the spindle assembly checkpoint (SAC), that delays mitosis, providing cells with additional time to resolve errors in microtubule-kinetochore attachment. ${ }^{22,23}$ The SAC inhibits the anaphase-promoting complex/ cyclosome $(\mathrm{APC} / \mathrm{C})$, an E3-ubiquitin ligase that triggers mitotic exit mostly by targeting cyclin $\mathrm{B}$ for degradation, thus inhibiting Cdk1 activity. The SAC-dependent delay in mitosis increases the susceptibility of cells to undergo cell death. Alternatively, cells can escape from the mitotic arrest by slowly degrading cyclin B, a process known as mitotic slippage (see below; Figure 2).

Regardless of their effectiveness in cancer treatment, microtubule-targeted drugs exhibit important side effects. Patients treated with these drugs develop severe myelosuppression derived from proliferation impairment of cycling bone marrow cells. Neurotoxicity is also frequent due to the effects of these drugs on microtubules, which are basic components of neurons. In addition, patients treated with microtubule poisons often acquire resistance to these drugs. ${ }^{20}$ Thus, although inhibiting microtubule function has demonstrated a significant antitumor activity, the side effects of these drugs limit their use and have prompted to identify alternative antimitotic drugs.

New spindle targets. One of the key regulators of the spindle are kinesin motor proteins, which have crucial functions in spindle assembly, chromosome congression and segregation. ${ }^{24} \mathrm{Eg} 5$ is a plus end-directed motor protein

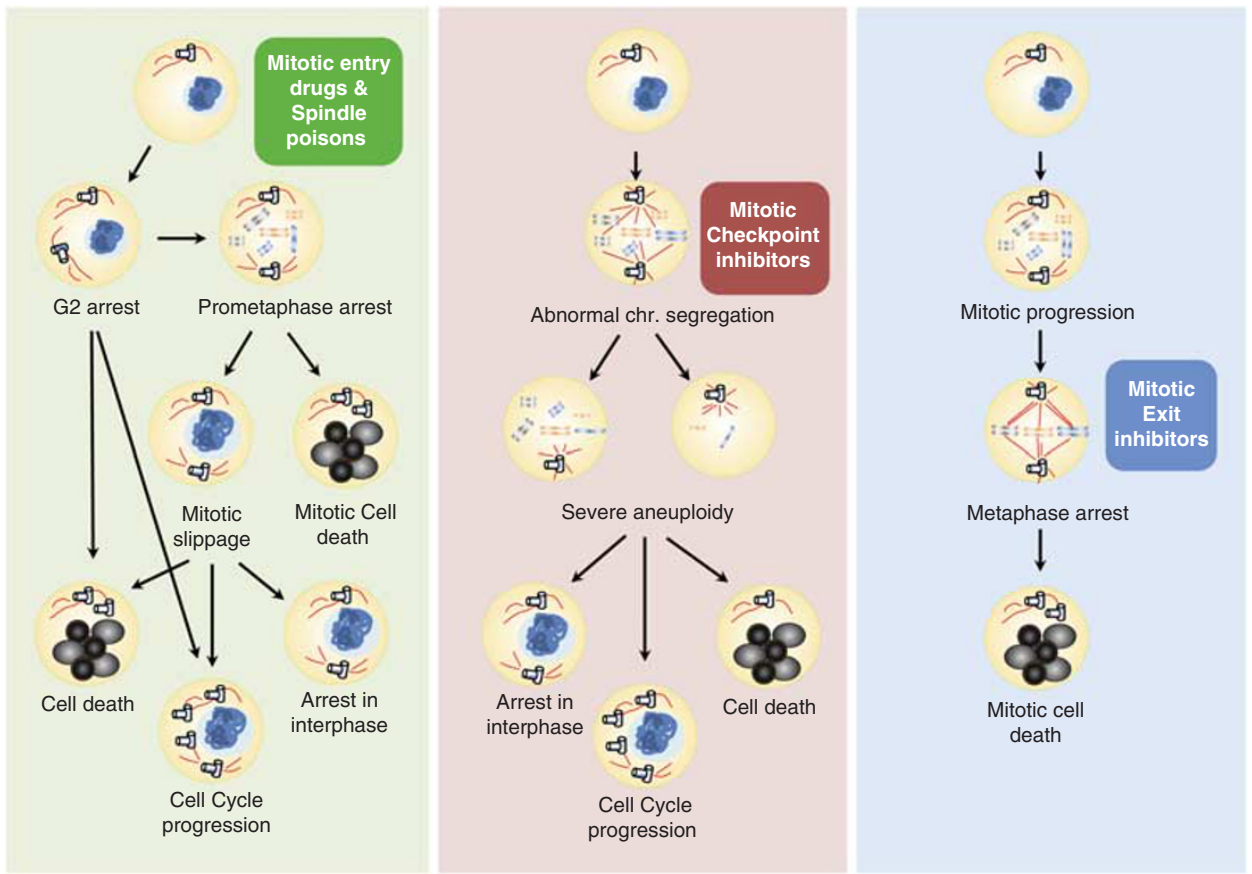

Figure 2 Models for targeting mitosis as an anticancer strategy. Treatment with mitotic entry inhibitors or spindle poisons leads to G2 or prometaphase arrest that eventually may end up in apoptosis during interphase or mitosis. However, cells may escape entering into a new cell cycle. Depending of the status of genes such as $p 53, p R b$ or p38, these cells may arrest before entering S phase, die or continue proliferating. Targeting mitotic checkpoint regulators, such as BubR1, Mps1 or Mad2, leads to severe levels of aneuploidy. Cells containing numerical chromosome aberrations can similarly either arrest in the subsequent G1 phase, progress through the cell cycle or undergo cell death. Inhibiting mitotic exit (e.g., by targeting APC/C-Cdc20) provokes a permanent metaphase arrest by preventing cyclin B1 degradation, thus irreversibly leading to mitotic cell death 
Table 1 Representative mitotic targets and inhibitors in clinical or preclinical studies (see references ${ }^{32,42,56,61,98,99}$ for extensive reviews on specific targets)

\begin{tabular}{|c|c|c|c|c|}
\hline Target & Activity & Inhibitors & $\begin{array}{l}\text { Clinical } \\
\text { phase }\end{array}$ & Tumor types \\
\hline \multicolumn{5}{|l|}{ Clinical stage } \\
\hline Aurora $A$ & Bipolar spindle formation & MLN-8237, MLN-8054 & $I-I I$ & $\begin{array}{l}\text { Melanoma, hematopoietic malignancies, } \\
\text { ovarian, breast and prostate carcinoma }\end{array}$ \\
\hline Aurora B & $\begin{array}{l}\text { Chromosome alignment } \\
\text { and mitotic checkpoint }\end{array}$ & GSK1070916A, AZD1152 & I & $\begin{array}{l}\text { Advanced solid tumors and acute } \\
\text { myeloid leukemia }\end{array}$ \\
\hline Cdk1 & Mitotic entry and progression & P276-00, EM-1421 & I-II & $\begin{array}{l}\text { Hematopoietic malignancies, breast } \\
\text { cancer, melanoma }\end{array}$ \\
\hline CENP-E & $\begin{array}{l}\text { Spindle dynamics and } \\
\text { mitotic checkpoint }\end{array}$ & GSK923295A & I & Acute lymphoblastic leukemia \\
\hline Eg5 & Spindle dynamics & Ispinesib, AZD4877, ARRY-520 & $I-I I$ & $\begin{array}{l}\text { Hematopoietic malignancies, bladder } \\
\text { cancer and advanced solid tumors }\end{array}$ \\
\hline Plk1 & Bipolar spindle formation & $\begin{array}{l}\text { GSK461364, TKM-080301, } \\
\text { NMS-1286937, BI6727, ON01910 }\end{array}$ & $I-I I$ & $\begin{array}{l}\text { Advanced or metastatic solid tumors } \\
\text { and hematopoietic malignancies }\end{array}$ \\
\hline \multicolumn{5}{|l|}{ Preclinical stage } \\
\hline APC/C (Cdc20) & Mitotic E3-ubiquitin ligase & TAME & None & \\
\hline Mps1 & Mitotic checkpoint & NMS-P715, reversine, Mps1 IN-1 & None & \\
\hline
\end{tabular}

required for centrosome separation and thereby for building a bipolar spindle. ${ }^{25,26}$ The growing interest of this kinesin as an antitumor target emerged from the pioneer identification of monastrol, the first small-molecule inhibitor of Eg5. ${ }^{27}$ Inhibition of Eg5 activity generates monopolar spindles that lead to mitotic arrest in a SAC-dependent manner. ${ }^{26,27}$ This mitotic arrest culminates in cell death in a broad range of tumor cell lines, both in cell culture and xenografts, ${ }^{28,29}$ and taxane-resistant cancer cells remain sensitive to Eg5 inhibition. ${ }^{30}$ As a consequence of these promising results, a number of Eg5 inhibitors have been studied, and some of them have recently entered into clinical trials ${ }^{31}$ (Table 1). Although side effects are moderate, the clinical efficacy of Eg5 inhibitors has been limited, with only few studies demonstrating a partial response. ${ }^{32}$

In addition to Eg5, kinesin CENP-E has recently emerged as a potential antitumoral target. This kinesin is also a plus end-directed motor protein that appears to have a pivotal role specifically in mitosis. CENP-E stabilizes the interactions between microtubules and kinetochores of the mitotic spindle ${ }^{33}$ and regulates the mitotic checkpoint by modulating the function of BubR1. ${ }^{34}$ CENP-E is an essential mammalian gene, and its complete inhibition leads to defective mitosis with unaligned chromosomes and apoptosis. ${ }^{35,36}$ Partial inactivation of CENP-E has exhibited antitumor activity in xenografted human tumors, as well as in chemically-induced tumors in mice. ${ }^{36,37}$ Farnesyl transferase inhibitors that hamper CENP-E activity and specific smallmolecule inhibitors of CENP-E are currently in phase I of clinical trials ${ }^{32,38}$ (Table 1).

In addition to kinesins, mitotic kinases such as Aurora $A$ and Plk1 are also major regulators of spindle formation. ${ }^{16,39,40}$ These two mitotic kinases are overexpressed in cancer cells, often correlating with worse prognosis, and their inhibition results in mitotic defects, making them attractive mitotic targets. ${ }^{41,42}$ As previously reported for Eg5, disruption of Plk1 or Aurora A activity results in SAC-dependent mitotic arrest as a consequence of monopolar spindle formation, and this arrest correlates with increased apoptosis in multiple cancer cell lines. Several ATP-competitive compounds specific against these kinases have already entered into clinical trials showing partial responses in specific tumors ${ }^{42-44}$ (Table 1). Further efforts are now dedicated to identify more specific compounds directed against the polo-box domain instead of the kinase domain of Plk1. ${ }^{44}$ Similarly to microtubule poisons, inhibition of these targets may result in apoptotic cell death or the generation of tetraploid cells through mitotic slippage. These tetraploid cells may be unable to replicate DNA in the following cell cycle if the corresponding checkpoints are functional or could eventually proliferate as polyploid cultures (Figure 2).

\section{Targeting the Mitotic Checkpoint}

To prevent chromosome missegregation, the SAC delays the metaphase-anaphase transition until all chromosomes are properly attached to microtubules. ${ }^{23}$ Abrogating the SAC therefore results in defective or abnormal chromosome segregation, generating polyploid or aneuploid cells (Box 1). It has been suggested that a partially compromised mitotic checkpoint may lead to chromosomal instability (CIN), therefore promoting tumorigenesis. Mice heterozygous for SAC components, such as Mad2 or BubR1, are prone to develop spontaneous tumors after long latencies. ${ }^{45,46}$ In addition, mice with a weakened checkpoint due to partial loss of Bub3 or BubR1 also exhibit high rates of tumorigenesis after carcinogenic treatment. ${ }^{47,48}$ Although these findings suggest a direct link between chromosome instability and tumorigenesis, mutations in mitotic checkpoint genes are not frequent in human tumors. ${ }^{49}$ This is not surprising taking into account the lethal phenotype observed after the complete abrogation of these genes in engineered mouse models. ${ }^{50}$

As most tumor cells are aneuploid or display certain levels of CIN (Box 1), it has been proposed that abrogating the SAC may induce further instability and may be therefore used as a therapeutic strategy. Genetic elimination of Mad2 or BubR1 results in widespread chromosome missegregation that trigger programmed cell death. ${ }^{45,46,48,51}$ In contrast to other cell cycle checkpoints, the SAC is essential for cell survival, and targeting this mitotic checkpoint could constitute 
Box 1 Tumor-associated aneuploidy

Aneuploidy, or the abnormal number of chromosomes that differ from multiples of the normal ploidy, is a hallmark of cancer cells. About $90 \%$ of solid tumors and $85 \%$ of hematopoietic neoplasias are aneuploid. ${ }^{49}$ A possible cause of aneuploidy is chromosomal instability (CIN), an increased susceptibility to gain or loss of chromosomes, resulting in unstable karyotypes. However, many aneuploid tumors are known to stably maintain their karyotypes, and both concepts, although frequently mixed in the literature, have different implications in cancer. On one hand, the gene dosage variation resulting from $\mathrm{CIN}$ may provide proliferative advantages by increasing the mutation rate in oncogenes, tumor suppressors or tumor susceptibility genes. Recent data suggest that inactivation of major tumor suppressor pathways, such as $\mathrm{pRb}$ or p53, leads to a mitotic stress due to the overexpression of the SAC regulator Mad2, suggesting that CIN may be an early, common event in tumor cells. ${ }^{91,92}$ On the other hand, the induction of strong and acute CIN (e.g., by SAC abrogation) may be detrimental for cell viability, and this is now considered as a possible therapeutic strategy (see figure and main text). The relevance of stable aneuploidies for cancer development of therapy is more complex. Studies in yeast and primary mouse fibroblasts have shown that just one extra chromosome may result in important energetic and metabolic aberrations. These alterations are likely to result from the additional load of proteins encoded by the extra chromosomes, leading to important problems in proliferation and enhanced cell lethality. ${ }^{93,94}$ Other studies, however, suggest that aneuploidy facilitates phenotypic variation in yeast, providing proliferative advantages upon suboptimal conditions. ${ }^{95} \mathrm{~A}$ recent study has shown that small molecules synergize with proteotoxic and energy stress efficiently and specifically antagonize the proliferation of aneuploid cells. ${ }^{96}$ All together, these studies suggest that $\mathrm{CIN}$ or aneuploidy may be detrimental for cell proliferation and lead to a proliferative stress, resulting in cell cycle arrest or apoptosis (see figure). In those cases where CIN or aneuploidy results in proliferative advantages, tumor cells may be specifically sensitive to SAC abrogation (which may induce lethal levels of instability) or specific compounds targeting the energetic and proteotoxic stress pathways. ${ }^{97}$
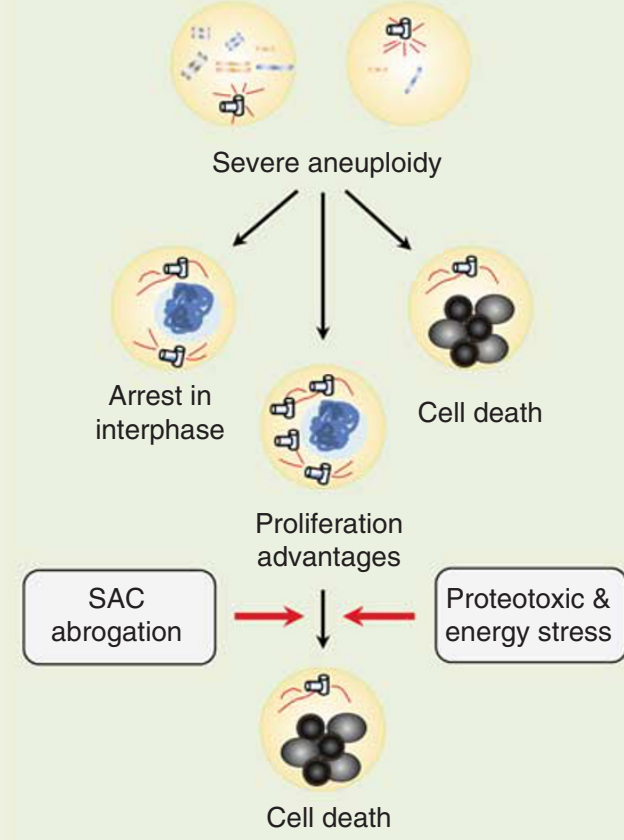

Cell death a novel strategy to kill tumor cells by inducing lethal instability. ${ }^{22,52,53}$ Inhibition of mitotic checkpoint activity by depletion of Mad2 or BubR1 provokes severe chromosome segregation errors that are incompatible with human cell viability. ${ }^{54,55}$ In addition, reducing protein levels of BubR1 or Mps1, another protein kinase involved in the SAC, sensitizes tumor cells to taxol. ${ }^{53}$ Checkpoint abrogation by depletion of Mad2 or chemical inhibition of Mps1 have been evaluated as antitumor strategies in xenografted human tumors. ${ }^{56,57}$ Aurora B may also be considered as a SAC target, as its downregulation overcomes the arrest in the presence of microtubule-stabilizing agents such as taxol. ${ }^{39,58}$ Aurora B is required for embryo viability, and its genetic or chemical inhibition results in the generation of tetraploid cells with reduced viability, suggesting its relevance as a therapeutic target. $^{59,60}$

Interestingly, compared with non-transformed cells, cancer cell lines are more sensitive to undergo cell death in response to checkpoint abrogation. ${ }^{52,61}$ Whether this is a consequence of the abnormal number of chromosomes commonly found in tumor cells (Box 1) is not well established. It has been proposed that, as a consequence of the extra chromosomes, tumor cells may require more time to properly align chromosomes at metaphase. ${ }^{62}$ This time requirement would make tumor cells more prone to chromosome missegregation and thereby more sensitive to die because of checkpoint abrogation. Other possible, although less explored, explanations for the increased susceptibility to the induction of CIN include differences in gene dosage or synthetic lethal interactions with deregulated pathways. Given the difficulties of reaching a complete inhibition of the mitotic checkpoint in vivo, a combination with sublethal doses of microtubule poisons has been suggested to achieve effective antitumor activities in clinical settings. ${ }^{53}$

\section{Targeting Mitotic Exit}

Mitotic slippage is thought to be one of the main mechanisms of resistance against antimitotic drugs ${ }^{63-65}$ (Figure 2). During a normal cell cycle, mitotic exit is driven by APC/C-Cdc20dependent cyclin $\mathrm{B} 1$ degradation. Inhibiting mitotic progression triggers the SAC and inhibits APC/C-Cdc20. However, this inhibition is transient and slow, and progressive degradation of cyclin B1 may happen despite the unsatisfaction of the mitotic checkpoint. ${ }^{64,65}$ Cells that escape from mitotic cell death can either die at the following cell cycle stage, arrest in a tetraploid state or undergo several rounds of division, ${ }^{63}$ depending of the status of genes such as p53 or p38 (Lanni et al. ${ }^{66}$; Rieder et al. ${ }^{67}$; Figure 2). Inhibition of apoptosis increases mitotic slippage, ${ }^{64,68}$ suggesting that both processes are somehow linked (see below). Remarkably, several independent studies have observed no correlation between cell death and mitotic duration, suggesting that cell fate after treatment with antimitotic drugs is not exclusively dictated by mitotic length. ${ }^{65}$ Gascoigne and Taylor proposed an elegant model based on two competing networks, one that involves mitotic cell death pathways and other that regulates cyclin B1 degradation. These two networks have specific thresholds that compete during mitotic arrest. If cyclin B1 levels fall under a certain threshold before caspase activation has reached its 
cell death threshold, then cells will exit from mitosis. On the other hand, if cyclin B1 levels fall slowly, then cell death threshold will be achieved first and cells will die in mitosis. ${ }^{69}$

According with this model, it is predictable that completely blocking mitotic exit might be a powerful strategy to induce mitotic cell death. We have recently demonstrated that impairing cyclin B1 degradation in vivo by genetic elimination of Cdc20 is much more efficient in killing tumor cells than targeting the spindle assembly with classical poisons or new drugs inhibiting Eg5 or Plk1. ${ }^{70}$ These observations are in agreement with the pioneer results using RNA interference (RNAi) against Cdc20 or expressing a non-degradable mutant of cyclin B1 in several tumor cell lines. ${ }^{71}$ Whereas classical antimitotic drugs (vincristine or taxol) and new available mitotic inhibitors against Plk1 or kinesin Eg5 (BI2536 or monastrol, respectively) only induce a partial response in aggressive tumors, Cdc20 ablation results in complete metaphase arrest, leading to massive apoptotic cell death and complete tumor elimination in vivo. ${ }^{70}$ Importantly, mitotic exit inhibition also affects pRb-null, p53-null or SAC-deficient cells, ${ }^{70,71}$ suggesting widespread uses. In vitro, Cdc20-null tumor cells cannot escape from the metaphase arrest and die of mitotic cell death in $6-30 \mathrm{~h}^{70}$ Altogether, these results suggest that drugs that target Cdc20 or other components involved in cyclin B1 degradation, such as core components of the APC/C or the proteasome, could be quite effective in killing tumor cells. Indeed, a genome-wide RNAi screen has recently identified synthetic lethal interaction between K-RAS oncogenic signaling and the inhibition of core subunits of the APC/C, such as APC1 and APC4 ${ }^{72}$. These results have been reinforced with the discovery of tosyl-L-arginine methyl ester (TAME), an APC/C small-molecule inhibitor that efficiently arrests tumor cells in mitosis triggering cell death. ${ }^{73}$

Although targeting mitotic exit may be highly efficient, how can this strategy discriminate between tumor and normal proliferating cells? As previously suggested, APC/C-Cdc20 inhibitors could be useful in combination with other mitotic poisons by slowing cyclin B1 degradation and thus providing enough time to reach the apoptotic threshold of tumor cells. ${ }^{67}$ Other strategies to consider are those orientated to protect normal proliferating cells by impairing their entry into mitosis. Some studies proposed to use Cdk2/4 inhibitors to arrest pRbproficient cells in G0/G1 and protect them from mitotic exit inhibitors. In contrast, pRb-null tumor cells would overcome this arrest, thereby making them sensitive to arrest and die in mitosis. ${ }^{74}$ Other studies using xenotransplanted tumors have demonstrated that prolonged fasting dramatically reduces the toxic side effects of chemotherapy, while maintaining its efficacy against tumor cells. ${ }^{75}$ All these strategies could help to improve the therapeutic window of antimitotic therapies, making them tumor selective.

An interesting question that rises from these studies is whether tumors cells treated with APC/C inhibitors may adapt to this situation and what would be the molecular requirements for this resistance. In our previous work, we have shown that kinases Cdk1 and Mastl are required for Cdc20dependent metaphase arrest and apoptosis. Cdk1 and Mastl inhibition strongly synergizes to promote exit from mitosis and tumor cell survival in vitro. ${ }^{70}$ Thus, whereas APC/C inhibition may enhance the therapeutic benefits of specific mitotic drugs, inhibition of Cdk1 or Mastl should be avoided. In summary, targeting mitotic exit offers effective possibilities for killing tumor cells, and further investigation will be required to identify druggable targets and to validate this therapeutic strategy in specific tumors in vivo.

\section{Molecular Pathways of Mitotic Cell Death}

Cells treated with antimitotic drugs exhibit a prometaphase delay, due to SAC activity, that is often followed by mitotic cell death. Although the link between mitotic arrest and apoptosis is well established, the molecular determinants of this apoptotic response are mostly unknown. For a long time, the activity of the mitotic checkpoint has been suspected to be required to kill tumor cells treated with spindle poisons. ${ }^{29}$ Consistent with these observations, the mitotic checkpoint protein BubR1 has shown proapoptotic activity in polyploid cells that escaped from prolonged mitotic arrest. ${ }^{76}$ However, studies using Cdc20-deficient cells, in which the mitotic checkpoint is satisfied, have proved that the mere SAC activity is not required for inducing mitotic cell death (Figure 2). Consequently, a proapoptotic signal independent of the SAC accumulates to trigger apoptosis during mitotic arrest. ${ }^{70,71}$ Recent data suggest that caspases are not required for the activity of the mitotic checkpoint or for mitotic slippage, ${ }^{77}$ suggesting that mitotic and death pathways are two independent processes. How these two processes talk to each other and how cell death is triggered and modulated during prolonged mitosis is a central question that needs to be answered.

Mitotic cell death generally occurs via intrinsic or mitochondrial apoptosis, which is regulated by the $\mathrm{Bcl}-2$ family of proteins. ${ }^{78}$ Prosurvival members, including $\mathrm{Bcl}-\mathrm{X}_{\mathrm{L}}, \mathrm{Bcl}-2$ and $\mathrm{Mcl}-1$, antagonize apoptosis by blocking the activity of proapoptotic regulators. On the other hand, proapoptotic members, Bax and Bak, function as mediators of mitochondrial membrane permeability, allowing the activation of caspases, the proteases that catalyze cellular death. Interestingly, cyclin B1-Cdk1 may have a central role in the regulation of these apoptotic proteins (Figure 3). Cdk1 has been shown to phosphorylate both $\mathrm{Bcl}-\mathrm{X}_{\mathrm{L}}$ and $\mathrm{Bcl}-2$, leading to the inactivation of their antiapoptotic activities. ${ }^{79}$ During a normal mitosis, transient activation of Cdk1 fails to reach the phosphorylation threshold that overrides the antiapoptotic activity of these regulators. However, if Cdk1 is active for too long, the threshold is reached, causing inactivation of the antiapoptotic function and eventual apoptosis. ${ }^{79}$ Recent studies have also elucidated a new role of Cdk1 in controlling the turnover of $\mathrm{Mcl}-1$. Phosphorylation of $\mathrm{Mcl}-1$ makes it sensitive to its polyubiquitination by the Skp-Cullin-F-box protein complex (SCF; Figure 3). Once Mcl-1 is phosphorylated by JNK, p38 and CKII kinases, it is recruited to the SCF by the tumor suppressor protein Fbw7 and degraded by the proteasome. During normal mitosis, phosphorylation of Mcl-1 is counteracted by phosphatases such as PP2A. However, upon extended mitosis, Cdk1 promotes T92 phosphorylation and PP2A dissociation of Mcl-1, allowing its Fbw7-dependent degradation. ${ }^{80} \mathrm{Mcl}-1$ has also been reported to be degraded by APC/C-Cdc20 when it is phosphorylated by Cdk1 in mitotic arrested cells. ${ }^{81}$ Thus, by promoting degradation of $\mathrm{Mcl}-1$ in 


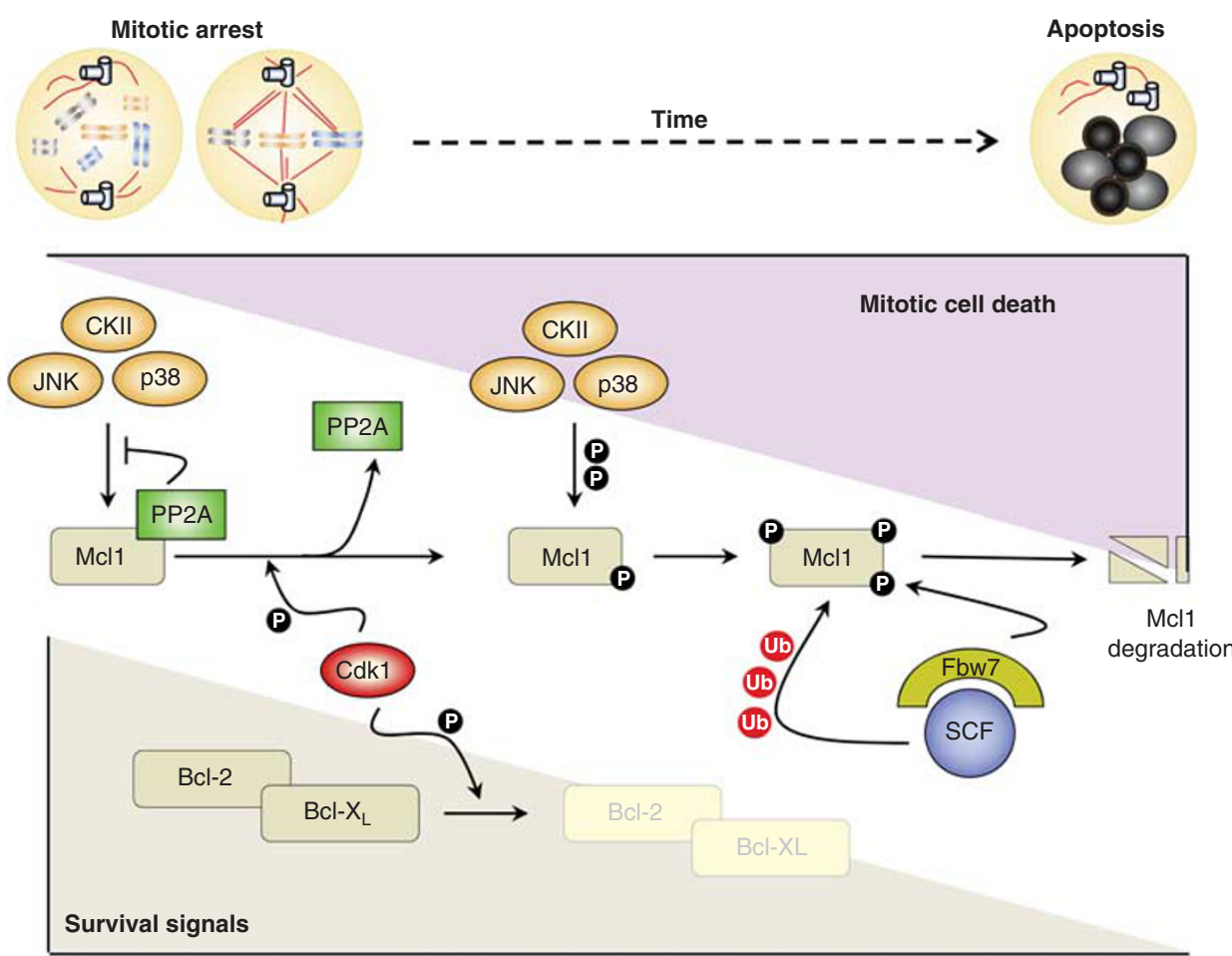

Figure 3 Mitotic cell death is governed by Cdk1 activity. During prolonged mitotic arrest (e.g., by using microtubule poisons, AurA or Plk1 inhibitors or inhibiting the APC/ C), Cdk1 activity regulates the stability of antiapoptotic effectors. Cdk1-dependent phosphorylation of Mcl-1 allows its phosphorylation by other kinases, such as JNK, p38 and CKII. Highly phosphorylated forms of Mcl-1 are recognized and ubiquitinated by the E3-ubiquitin ligase SCF, leading to its degradation by the proteasome $26 \mathrm{~S}$. On the other hand, phosphorylation of Bcl- $X_{L}$ and Bcl-2 by Cdk1 drives their conversion to an inactive form. Thus, degradation of Mcl-1 and inactivation of Bcl- $X_{L}$ and Bcl-2 lead to the loss of balance between pro- or antiapoptotic signals, resulting in mitotic cell death. Cdk1 may also prevent mitotic cell death by phosphorylating and inhibiting caspases (not shown), and the regulation of its pro- and antiapoptotic functions is not well understood (see main text)

mitotic arrested cells, Cdk1 allows the activation of Bax and Bak proteins to promote mitotic cell death. Furthermore, the lengthened activation of Cdk1 may increase the activity of p53, which causes the transcription of proapoptotic proteins such as Bax and Puma, thereby initiating mitochondrial apoptosis. $^{82}$ In contrast, Cdk1 may also phosphorylate caspase- 9 and caspase-8, hampering their ability to trigger the intrinsic or extrinsic apoptotic pathways, respectively. ${ }^{83,84}$ Altogether, these data suggest that cyclin B1-Cdk1 complexes have a key role in balancing survival and cell death pathways that dictate the fate of cells during mitotic arrest. How the proapoptotic and prosurvival activities of Cdk1 are controlled is not clear at present.

The therapeutic relevance of targeting the apoptotic machinery has already been shown by using $\mathrm{BH} 3$ mimetics. These compounds function as inhibitors of prosurvival members of the $\mathrm{Bcl}-2$ family and have demonstrated efficacy against either tumor cell lines or xenografted human tumors. ${ }^{85}$ In addition, BH3 mimetics increase the efficacy of microtubule poisons, suggesting a role for Bcl-2 proteins in mitotic cell death. ${ }^{86}$ Although apoptosis seems to be the main mechanism by which cells die after an extended mitotic arrest, the fact that caspase inhibitors, such as Z-VAD.fmk, or Bcl-2 overexpression fail to completely prevent cell death induced by treatment with spindle poisons has led to conclude that other mechanisms may have a role in mitotic cell death. ${ }^{71,87}$ From a molecular point of view, mitotic cell death may differ from classical apoptosis in the activation of a particular mitochon- drial permeabilization pathway. During mitotic arrest, caspase-2 stimulates mitochondrial membrane permeabilization, facilitating the release of several caspase activators that finally lead to caspase-3 activation, one of the main death effectors in apoptosis. ${ }^{82}$ Bid cleavage has been proposed to be the main activity by which caspase- 2 induces mitochondrial permeability. ${ }^{88}$ However, Cdk1 also phosphorylates caspase2 , preventing its ability to trigger apoptosis, ${ }^{89}$ and how caspase- 2 is activated during mitotic arrest therefore remains unknown.

\section{Conclusions}

After several decades of research, our understanding of the regulation of mitosis is quite advanced, and it has allowed the identification and characterization of novel mitotic targets and strategies for clinical use. A significant number of smallmolecule inhibitors against Cdks, Plk1, Aurora kinases, Mps1, Eg5 or the APC/C are now available for preclinical or clinical studies (Table 1). The initial clinical trials with some of these compounds have confirmed efficacy in vivo, but in most cases worse than classical microtubule-targeting drugs. The basis for these differences are not clear, although we believe that our scarce knowledge of the in vivo requirements for some of these targets, the need for improvement in specificity and potency of the current compounds, and the lack of biomarkers to select patients may explain the limited effect observed so far. Given the fact that the majority of human tumors divide 
very slowly, compared with cultured cell lines and tumors in animal models, it has been speculated that the success of microtubule poisons might be given by its ability to target non-mitotic functions of microtubules. ${ }^{90}$ Irrespectively of the molecular details behind the success of these classical microtubule poisons, it is now clear that additional strategies, such as targeting the mitotic checkpoint or blocking mitotic exit, are emerging possibilities that deserve further exploration. Targeting CIN or aneuploidy is also being considered (Box 1), although further molecular and in vivo research are required to establish clear pathways and properly validate targets. The goal in most of these strategies is killing cancer cells. However, it is actually surprising how insufficient is our knowledge on the molecular pathways that modulate mitotic cell death and the differences between this type of death and other forms of apoptosis. Mitosis and apoptosis researchers need to combine efforts for moving forward in this direction in the future.

\section{Conflict of Interest}

The authors declare no conflict of interest.

Acknowledgements. The Cell Division and Cancer group of the CNIO is supported by the Association for International Cancer Research (AICR no. 08-0188), Foundation Ramón Areces, the OncoCycle Programme (S-BIO-02832006) from the Comunidad de Madrid, the Spanish Ministry of Science and Innovation (SAF2009-07973 and OncoBIO Consolider-Ingenio 2010 Programme (CSD2007-00017)) and the European Union Seventh Framework Programme (MitoSys project; HEALTH-F5-2010-241548).

1. Malumbres M, Barbacid M. Mammalian cyclin-dependent kinases. Trends Biochem Sci 2005; 30: 630-641.

2. Malumbres M, Barbacid M. To cycle or not to cycle: a critical decision in cancer. Nat Rev Cancer 2001; 1: 222-231.

3. Malumbres M, Pevarello $P$, Barbacid M, Bischoff JR. CDK inhibitors in cancer therapy: what is next? Trends Pharmacol Sci 2008; 29: 16-21.

4. Shapiro Gl. Cyclin-dependent kinase pathways as targets for cancer treatment. J Clin Oncol 2006; 24: 1770-1783.

5. Rane SG, Dubus P, Mettus RV, Galbreath EJ, Boden G, Reddy EP et al. Loss of Cdk4 expression causes insulin-deficient diabetes and Cdk4 activation results in beta-islet cell hyperplasia. Nat Genet 1999; 22: 44-52.

6. Ortega S, Prieto I, Odajima J, Martin A, Dubus P, Sotillo R et al. Cyclin-dependent kinase 2 is essential for meiosis but not for mitotic cell division in mice. Nat Genet 2003; 35: 25-31.

7. Malumbres M, Sotillo R, Santamaria D, Galan J, Cerezo A, Ortega S et al. Mammalian cells cycle without the D-type cyclin-dependent kinases Cdk4 and Cdk6. Cell 2004; 118: 493-504.

8. Miliani de Marval PL, Macias E, Rounbehler R, Sicinski P, Kiyokawa H, Johnson DG et al. Lack of cyclin-dependent kinase 4 inhibits c-myc tumorigenic activities in epithelial tissues. Mol Cell Biol 2004; 24: 7538-7547.

9. Malumbres M, Barbacid M. Is Cyclin D1-CDK4 kinase a bona fide cancer target? Cancer Cell 2006; 9: 2-4.

10. Puyol M, Martin A, Dubus P, Mulero F, Pizcueta $P$, Khan $G$ et al. A synthetic lethal interaction between K-Ras oncogenes and Cdk4 unveils a therapeutic strategy for nonsmall cell lung carcinoma. Cancer Cell 2010; 18: 63-73.

11. Santamaria D, Barriere $C$, Cerqueira A, Hunt $S$, Tardy $C$, Newton $K$ et al. Cdk1 is sufficient to drive the mammalian cell cycle. Nature $2007 ; 448: 811-815$.

12. Bryant HE, Schultz N, Thomas HD, Parker KM, Flower D, Lopez E et al. Specific killing of BRCA2-deficient tumours with inhibitors of poly(ADP-ribose) polymerase. Nature 2005; 434: 913-917.

13. Johnson N, Li YC, Walton ZE, Cheng KA, Li D, Rodig SJ et al. Compromised CDK1 activity sensitizes BRCA-proficient cancers to PARP inhibition. Nat Med 2011; 17: 875-882.

14. Mayes PA, Dolloff NG, Daniel CJ, Liu JJ, Hart LS, Kuribayashi K et al. Overcoming hypoxia-induced apoptotic resistance through combinatorial inhibition of GSK-3\{beta\} and CDK1. Cancer Res 2011; 71: 5265-5275.
15. Goga A, Yang D, Tward AD, Morgan DO, Bishop JM. Inhibition of CDK1 as a potential therapy for tumors over-expressing MYC. Nat Med 2007; 13: 820-827.

16. Malumbres M. Physiological relevance of cell cycle kinases. Physiol Rev 2011; 91: 973-1007.

17. Goldberg ML. Greatwall kinase protects mitotic phosphosites from barbarian phosphatases. Proc Natl Acad Sci USA 2010; 107: 12409-12410.

18. Burgess A, Vigneron S, Brioudes E, Labbe JC, Lorca T, Castro A. Loss of human Greatwall results in G2 arrest and multiple mitotic defects due to deregulation of the cyclin B-Cdc2/ PP2A balance. Proc Natl Acad Sci USA 2010; 107: 12564-12569.

19. Voets $E$, Wolthuis RM. MASTL is the human orthologue of Greatwall kinase that facilitates mitotic entry, anaphase and cytokinesis. Cell Cycle 2010; 9: 3591-3601.

20. Jordan MA, Wilson L. Microtubules as a target for anticancer drugs. Nat Rev Cancer 2004; 4: 253-265.

21. Dumontet C, Jordan MA. Microtubule-binding agents: a dynamic field of cancer therapeutics. Nat Rev Drug Discov 2010; 9: 790-803.

22. Kops GJ, Weaver BA, Cleveland DW. On the road to cancer: aneuploidy and the mitotic checkpoint. Nat Rev Cancer 2005; 5: 773-785.

23. Musacchio A, Salmon ED. The spindle-assembly checkpoint in space and time. Nat Rev Mol Cell Biol 2007; 8: 379-393.

24. Wordeman L. How kinesin motor proteins drive mitotic spindle function: Lessons from molecular assays. Semin Cell Dev Biol 2010; 21: 260-268.

25. Sawin KE, LeGuellec K, Philippe M, Mitchison TJ. Mitotic spindle organization by a plusend-directed microtubule motor. Nature 1992; 359: 540-543.

26. Blangy A, Lane HA, d'Herin P, Harper M, Kress M, Nigg EA. Phosphorylation by p34cdc2 regulates spindle association of human $\mathrm{Eg} 5$, a kinesin-related motor essential for bipolar spindle formation in vivo. Cell 1995; 83: 1159-1169.

27. Mayer TU, Kapoor TM, Haggarty SJ, King RW, Schreiber SL, Mitchison TJ. Small molecule inhibitor of mitotic spindle bipolarity identified in a phenotype-based screen. Science 1999; 286: 971-974.

28. Sakowicz R, Finer JT, Beraud C, Crompton A, Lewis E, Fritsch A et al. Antitumor activity of a kinesin inhibitor. Cancer Res 2004; 64: 3276-3280.

29. Tao W, South VJ, Zhang Y, Davide JP, Farrell L, Kohl NE et al. Induction of apoptosis by an inhibitor of the mitotic kinesin KSP requires both activation of the spindle assembly checkpoint and mitotic slippage. Cancer Cell 2005; 8: 49-59.

30. Marcus Al, Peters U, Thomas SL, Garrett S, Zelnak A, Kapoor TM et al. Mitotic kinesin inhibitors induce mitotic arrest and cell death in Taxol-resistant and -sensitive cancer cells. J Biol Chem 2005; 280: 11569-11577.

31. Janssen A, Medema RH. Mitosis as an anti-cancer target. Oncogene 2011; 30: 2799-2809.

32. Huszar D, Theoclitou ME, Skolnik J, Herbst R. Kinesin motor proteins as targets for cancer therapy. Cancer Metastasis Rev 2009; 28: 197-208.

33. McEwen BF, Chan GK, Zubrowski B, Savoian MS, Sauer MT, Yen TJ. CENP-E is essential for reliable bioriented spindle attachment, but chromosome alignment can be achieved via redundant mechanisms in mammalian cells. Mol Biol Cell 2001; 12: 2776-2789.

34. Mao Y, Abrieu A, Cleveland DW. Activating and silencing the mitotic checkpoint through CENP-E-dependent activation/inactivation of BubR1. Cell 2003; 114: 87-98.

35. Putkey FR, Cramer T, Morphew MK, Silk AD, Johnson RS, McIntosh JR et al. Unstable kinetochore-microtubule capture and chromosomal instability following deletion of CENPE. Dev Cell 2002; 3: 351-365.

36. Wood KW, Lad L, Luo L, Qian X, Knight SD, Nevins $\mathrm{N}$ et al. Antitumor activity of an allosteric inhibitor of centromere-associated protein-E. Proc Natl Acad Sci USA 2010; 107: 5839-5844.

37. Weaver BA, Silk AD, Montagna C, Verdier-Pinard P, Cleveland DW. Aneuploidy acts both oncogenically and as a tumor suppressor. Cancer Cell 2007; 11: 25-36.

38. Schafer-Hales K, laconelli J, Snyder JP, Prussia A, Nettles JH, El-Naggar A et al. Farnesyl transferase inhibitors impair chromosomal maintenance in cell lines and human tumors by compromising CENP-E and CENP-F function. Mol Cancer Ther 2007; 6: 1317-1328.

39. Carmena M, Earnshaw WC. The cellular geography of aurora kinases. Nat Rev Mol Cell Biol 2003; 4: 842-854.

40. Barr FA, Sillje HH, Nigg EA. Polo-like kinases and the orchestration of cell division. Nat Rev Mol Cell Biol 2004; 5: 429-440.

41. Gautschi O, Heighway J, Mack PC, Purnell PR, Lara PN Jr, Gandara DR. Aurora kinases as anticancer drug targets. Clin Cancer Res 2008; 14: 1639-1648.

42. Lens SM, Voest EE, Medema RH. Shared and separate functions of polo-like kinases and aurora kinases in cancer. Nat Rev Cancer 2010; 10: 825-841.

43. Manfredi MG, Ecsedy JA, Meetze KA, Balani SK, Burenkova O, Chen W et al. Antitumor activity of MLN8054, an orally active small-molecule inhibitor of Aurora A kinase. Proc Natl Acad Sci USA 2007; 104: 4106-4111.

44. Perez de Castro I, de Carcer G, Montoya G, Malumbres M. Emerging cancer therapeutic opportunities by inhibiting mitotic kinases. Curr Opin Pharmacol 2008; 8: 375-383.

45. Michel LS, Liberal V, Chatterjee A, Kirchwegger R, Pasche B, Gerald W et al. MAD2 haploinsufficiency causes premature anaphase and chromosome instability in mammalian cells. Nature 2001; 409: 355-359.

46. Baker DJ, Jeganathan KB, Cameron JD, Thompson M, Juneja S, Kopecka A et al. BubR1 insufficiency causes early onset of aging-associated phenotypes and infertility in mice. Nat Genet 2004; 36: 744-749.

47. Babu JR, Jeganathan KB, Baker DJ, Wu X, Kang-Decker N, van Deursen JM. Rae1 is an essential mitotic checkpoint regulator that cooperates with Bub3 to prevent chromosome missegregation. J Cell Biol 2003; 160: 341-353. 
48. Dai W, Wang Q, Liu T, Swamy M, Fang Y, Xie S et al. Slippage of mitotic arrest and enhanced tumor development in mice with BubR1 haploinsufficiency. Cancer Res 2004; 64: 440-445.

49. Weaver BA, Cleveland DW. Does aneuploidy cause cancer? Curr Opin Cell Biol 2006; 18 $658-667$.

50. Schvartzman JM, Sotillo R, Benezra R. Mitotic chromosomal instability and cancer: mouse modelling of the human disease. Nat Rev Cancer 2010; 10: 102-115.

51. Dobles M, Liberal V, Scott ML, Benezra R, Sorger PK. Chromosome missegregation and apoptosis in mice lacking the mitotic checkpoint protein Mad2. Cell 2000; 101: 635-645.

52. Stolz A, Vogel C, Schneider V, Ertych N, Kienitz A, Yu H et al. Pharmacologic abrogation of the mitotic spindle checkpoint by an indolocarbazole discovered by cellular screening efficiently kills cancer cells. Cancer Res 2009; 69: 3874-3883.

53. Janssen A, Kops GJ, Medema RH. Elevating the frequency of chromosome mis-segregation as a strategy to kill tumor cells. Proc Natl Acad Sci USA 2009; 106 19108-19113.

54. Kops GJ, Foltz DR, Cleveland DW. Lethality to human cancer cells through massive chromosome loss by inhibition of the mitotic checkpoint. Proc Natl Acad Sci USA 2004; 101: 8699-8704.

55. Michel L, Diaz-Rodriguez E, Narayan G, Hernando E, Murty VV, Benezra R. Complete loss of the tumor suppressor MAD2 causes premature cyclin B degradation and mitotic failure in human somatic cells. Proc Natl Acad Sci USA 2004; 101: 4459-4464.

56. Colombo R, Caldarelli M, Mennecozzi M, Giorgini ML, Sola F, Cappella P et al. Targeting the mitotic checkpoint for cancer therapy with NMS-P715, an inhibitor of MPS1 kinase. Cancer Res 2010; 70: 10255-10264.

57. Kaestner P, Bastians H. Mitotic drug targets. J Cell Biochem 2010; 111: 258-265.

58. Nezi L, Musacchio A. Sister chromatid tension and the spindle assembly checkpoint. Curr Opin Cell Biol 2009; 21: 785-795.

59. Girdler F, Gascoigne KE, Eyers PA, Hartmuth S, Crafter C, Foote KM et al. Validating Aurora B as an anti-cancer drug target. J Cell Sci 2006; 119: 3664-3675.

60. Fernandez-Miranda G, Trakala M, Martin J, Escobar B, Gonzalez A, Ghyselinck NB et al. Genetic disruption of aurora B uncovers an essential role for aurora $C$ during early mammalian development. Development 2011; 138: 2661-2672.

61. Kwiatkowski N, Jelluma N, Filippakopoulos P, Soundararajan M, Manak MS, Kwon M et al. Small-molecule kinase inhibitors provide insight into Mps1 cell cycle function. Nat Chem Biol 2011; 6: 359-368

62. Yang Z, Loncarek J, Khodjakov A, Rieder CL. Extra centrosomes and/or chromosomes prolong mitosis in human cells. Nat Cell Biol 2008; 10: 748-751.

63. Rieder CL, Maiato H. Stuck in division or passing through: what happens when cells cannot satisfy the spindle assembly checkpoint. Dev Cell 2004; 7: 637-651.

64. Brito DA, Rieder CL. Mitotic checkpoint slippage in humans occurs via cyclin $B$ destruction in the presence of an active checkpoint. Curr Biol 2006; 16: 1194-1200.

65. Gascoigne KE, Taylor SS. Cancer cells display profound intra- and interline variation following prolonged exposure to antimitotic drugs. Cancer Cell 2008; 14: 111-122.

66. Lanni JS, Jacks T. Characterization of the p53-dependent postmitotic checkpoint following spindle disruption. Mol Cell Biol 1998; 18: 1055-1064.

67. Rieder CL, Medema RH. No way out for tumor cells. Cancer Cell 2009; 16: 274-275.

68. Brito DA, Rieder CL. The ability to survive mitosis in the presence of microtubule poisons differs significantly between human nontransformed (RPE-1) and cancer (U2OS, HeLa) cells. Cell Motil Cytoskeleton 2009; 66: 437-447.

69. Gascoigne KE, Taylor SS. How do anti-mitotic drugs kill cancer cells? J Cell Sci 2009; 122 (Pt 15): 2579-2585.

70. Manchado E, Guillamot M, de Carcer G, Eguren M, Trickey M, Garcia-Higuera I et al. Targeting mitotic exit leads to tumor regression in vivo: Modulation by Cdk1, Mastl, and the PP2A/B55alpha,delta phosphatase. Cancer Cell 2010; 18: 641-654.

71. Huang HC, Shi J, Orth JD, Mitchison TJ. Evidence that mitotic exit is a better cance therapeutic target than spindle assembly. Cancer Cell 2009; 16: 347-358.

72. Luo J, Emanuele MJ, Li D, Creighton CJ, Schlabach MR, Westbrook TF et al. A genomewide RNAi screen identifies multiple synthetic lethal interactions with the Ras oncogene. Cell 2009; 137: 835-848.

73. Zeng X, Sigoillot F, Gaur S, Choi S, Pfaff KL, Oh DC et al. Pharmacologic inhibition of the anaphase-promoting complex induces a spindle checkpoint-dependent mitotic arrest in the absence of spindle damage. Cancer Cell 2010; 18: 382-395.

74. Blagosklonny MV, Pardee AB. Exploiting cancer cell cycling for selective protection of normal cells. Cancer Res 2001; 61: 4301-4305.
75. Raffaghello L, Lee C, Safdie FM, Wei M, Madia F, Bianchi G et al. Starvation-dependent differential stress resistance protects normal but not cancer cells against high-dose chemotherapy. Proc Natl Acad Sci USA 2008; 105: 8215-8220.

76. Shin HJ, Baek KH, Jeon AH, Park MT, Lee SJ, Kang CM et al. Dual roles of human BubR1, a mitotic checkpoint kinase, in the monitoring of chromosomal instability. Cancer Cell 2003; 4: 483-497.

77. Lee $\mathrm{K}, \mathrm{Kenny} \mathrm{AE}$, Rieder $\mathrm{CL}$. Caspase activity is not required for the mitotic checkpoint or mitotic slippage in human cells. Mol Biol Cell 2011; 22: 2470-2479.

78. Letai AG. Diagnosing and exploiting cancer's addiction to blocks in apoptosis. Nat Rev Cancer 2008; 8: 121-132

79. Terrano DT, Upreti M, Chambers TC. Cyclin-dependent kinase 1-mediated Bcl-xL/Bcl-2 phosphorylation acts as a functional link coupling mitotic arrest and apoptosis. Mol Cell Biol 2010; 30: 640-656.

80. Wertz IE, Kusam S, Lam C, Okamoto T, Sandoval W, Anderson DJ et al. Sensitivity to antitubulin chemotherapeutics is regulated by MCL1 and FBW7. Nature 2011; 471: 110-114.

81. Harley ME, Allan LA, Sanderson HS, Clarke PR. Phosphorylation of Mcl-1 by CDK1-cyclin B1 initiates its Cdc20-dependent destruction during mitotic arrest. EMBO J 2010; 29: 2407-2420.

82. Castedo M, Perfettini JL, Roumier T, Andreau K, Medema R, Kroemer G. Cell death by mitotic catastrophe: a molecular definition. Oncogene 2004; 23: 2825-2837.

83. Allan LA, Clarke PR. Phosphorylation of caspase- 9 by CDK1/cyclin B1 protects mitotic cells against apoptosis. Mol Cell 2007; 26: 301-310.

84. Matthess Y, Raab M, Sanhaji M, Lavrik IN, Strebhardt K. Cdk1/cyclin B1 controls Fasmediated apoptosis by regulating caspase-8 activity. Mol Cell Biol 2010; 30: 5726-5740.

85. Oltersdorf T, Elmore SW, Shoemaker AR, Armstrong RC, Augeri DJ, Belli BA et al. An inhibitor of Bcl-2 family proteins induces regression of solid tumours. Nature 2005; 435 : 677-681.

86. Kutuk O, Letai A. Alteration of the mitochondrial apoptotic pathway is key to acquired paclitaxel resistance and can be reversed by ABT-737. Cancer Res 2008; 68: 7985-7994.

87. Vitale I, Galluzzi L, Castedo M, Kroemer G. Mitotic catastrophe: a mechanism for avoiding genomic instability. Nat Rev Mol Cell Biol 2011; 12: 385-392.

88. Bonzon C, Bouchier-Hayes L, Pagliari LJ, Green DR, Newmeyer DD. Caspase-2-induced apoptosis requires bid cleavage: a physiological role for bid in heat shock-induced death. Mol Biol Cell 2006; 17: 2150-2157.

89. Andersen JL, Johnson CE, Freel CD, Parrish AB, Day JL, Buchakjian MR et al. Restraint of apoptosis during mitosis through interdomain phosphorylation of caspase-2. EMBO $J$ 2009; 28: 3216-3227.

90. Komlodi-Pasztor E, Sackett D, Wilkerson J, Fojo T. Mitosis is not a key target of microtubule agents in patient tumors. Nat Rev Clin Oncol 2011; 8: 244-250.

91. Schvartzman JM, Duijf PH, Sotillo R, Coker C, Benezra R. Mad2 Is a critical mediator of the chromosome instability observed upon Rb and p53 pathway inhibition. Cancer Cell 2011; 19: 701-714.

92. Malumbres M. Oncogene-induced mitotic stress: p53 and pRb get mad too. Cancer Cell 2011; 19: 691-692.

93. Torres EM, Sokolsky T, Tucker CM, Chan LY, Boselli M, Dunham MJ et al. Effects of aneuploidy on cellular physiology and cell division in haploid yeast. Science 2007; 317: 916-924.

94. Williams BR, Prabhu VR, Hunter KE, Glazier CM, Whittaker CA, Housman DE et al. Aneuploidy affects proliferation and spontaneous immortalization in mammalian cells. Science 2008; 322: 703-709.

95. Pavelka N, Rancati G, Zhu J, Bradford WD, Saraf A, Florens L et al. Aneuploidy confers quantitative proteome changes and phenotypic variation in budding yeast. Nature 2010; 468: $321-325$.

96. Tang YC, Williams BR, Siegel JJ, Amon A. Identification of aneuploidy-selective antiproliferation compounds. Cell 2011; 144: 499-512.

97. Manchado E, Malumbres M. Targeting aneuploidy for cancer therapy. Cell 2011; 144 465-466.

98. Zeng X. Pharmacologic inhibition of the anaphase-promoting complex induces a spindle checkpoint-dependent mitotic arrest in the absence of spindle damage. Cancer Cell 2010; 18: $382-395$.

99. Malumbres M, Barbacid M. Cell cycle, CDKs and cancer: a changing paradigm. Nat Rev Cancer 2009: 9: 153-166. 